\section{DGI-045 MANAGEMENT OF THE HAEMATOLOGICAL TOXICITY INDUCED BY BENDAMUSTINE}

doi:10.1136/ejhpharm-2013-000276.311

A Bosó-Ribelles, A Morego-Soler, B Arribas-Diaz, JC Titos-Arcos, AM Rizo-Cerdá, P Selvi-Sabater, MC Sánchez-Mulero, I Sánchez-Martinez, N Manresa-Ramón, J LeónVillar. Hospital Morales Meseguer, Hospital Pharmacy, Murcia, Spain

Background Bendamustine is approved in Spain for the treatment of chronic lymphocytic leukaemia (CLL), Non Hodgkin Lymphoma (NHL) and multiple myeloma (MM). The most frequent adverse reactions are haematological. Usually patients require supportive treatment with granulocyte colony-stimulating factors (G-CSF) for neutropenia and erythropoietins for anaemia.

Purpose To describe the approach to neutropenia and anaemia caused by bendamustine in patients diagnosed with NHL, CLL and MM in our Hospital

Materials and Methods Descriptive and retrospective study of patients treated with bendamustine between November 2008 and February 2012 in our hospital. We collected data on age, sex, diagnosis, neutrophils count and haemoglobin before treatment and after receiving bendamustine, the proportion of patients requiring G-CSF (filgrastim or pegfilgrastim) or erythropoietins (darbepoetin alfa) Average number of G-CSF and erythropoietins doses.

Results A total of 38 patients received bendamustine, of whom 13 were women and 25 were men, with a mean age of 67 years old. 28 patients were diagnosed with NHL, 4 with MM and 6 with LLC. Before treatment, the neutrophils count was $4,846 / \mathrm{mm}^{3}$ and haemoglobin $11.7 \mathrm{~g} / \mathrm{dL}$. Later these figures were $2,440 / \mathrm{mm}^{3}$ for neutrophils and haemoglobin $11 \mathrm{~g} / \mathrm{dl} .73 .7 \%$ of patients required G-CSF and $10.5 \%$ erythropoietins. The median number of doses of G-CSF and darbepoetin alfa respectively were 6 and 2.5

Conclusions Bendamustine appears well tolerated. Supportive treatment with G-CSF is required in the majority of patients to maintain neutrophil count. This is not the case for anaemia, which occurs less frequently, requiring less rescue treatment. However these patients require close monitoring during treatment.

No conflict of interest.

\section{DGI-046 MONITORING OF ADHERENCE TO TREATMENT AND ADVERSE EVENTS IN THE MANAGEMENT OF PATIENTS WITH HIV INFECTION}

doi:10.1136/ejhpharm-2013-000276.312

'L Appolloni, 'F Locchi, ' 2 L Calza, ${ }^{2} \mathrm{~V}$ Colangeli, ${ }^{2} \mathrm{R}$ Manfredi, ${ }^{2} \mathrm{E}$ Magistrelli, ${ }^{2} \mathrm{~F}$ Piro, ${ }^{\prime} \mathrm{G}$ Papa, ${ }^{2} \mathrm{P}$ Viale, ' $\mathrm{C}$ Puggioli. 'Clinical Pharmacy, S. Orsola-Malpighi Hospital University of Bologna, Bologna, Italy; 'Infectious Diseases, S. Orsola-Malpighi Hospital University of Bologna, Bologna, Italy

Background Highly active antiretroviral treatment (HAART) is associated with improved health outcomes for people living with HIV/AIDS. Successful long-term treatment of HIV/AIDS requires near-perfect adherence to HAART. Constant monitoring of adherence to HAART and evaluation of related adverse events are two essential aspects for optimal management of patients with HIV.

Purpose To monitor adherence to antiretroviral treatment and adverse events of the outpatients of an HIV referral centre (department of Clinical Infectious Diseases, Policlinico S.Orsola-Malpighi, Bologna).

Materials and Methods The pharmacist was introduced in the department of Clinical Infectious Diseases in order to distribute the antiretroviral drugs and give information on the proper storage, use and possible interactions associated with the treatment. The pharmacist gives out an adherence questionnaire (10 questions about adherence, co-administered drugs and adverse events) to each patient to complete and return during the following visit. This information was entered into a database (Access) and the adherence to treatment and incidence of adverse events was calculated.

Results We analysed the adherence questionnaires of 659 patients, $74 \%$ of whom reported $100 \%$ adherence to treatment. Coadministered medicines may lead to poorer HAART adherence: patients taking polypharmacy showed medium-low adherence to treatment. Adherence was found to correlate inversely with the daily pill burden.

In terms of adverse effects, we developed a pharmacovigilance system, reporting 15 adverse drug reactions, $27 \%$ of which were rated severe. We analysed physical changes, gastrointestinal disorders and neuropsychiatric symptoms associated with the following regimens: efavirenz/emtricitabine/tenofovir, emtricitabine/tenofovir + atazanavir/ritonavir, efavirenz/emtricitabine/tenofovir, emtricitabine/tenofovir + atazanavir/ritonavir, emtricitabine/tenofovir + darunavir/ritonavir, abacavir/lamivudine + emtricitabine/tenofovir + darunavir/ritonavir, abacavir/lamivudine + atazanavir/ritonavir, abacavir/lamivudine + darunavir/ritonavir. Our results showed that the regimens with darunavir correlated with a lower incidence of side effects and perception of physical changes.

Conclusions The physician-pharmacist collaboration is an important support in monitoring adherence and adverse events related to HAART and contributes significantly to the optimal management of patients with HIV infection.

No conflict of interest.

\section{DGI-047 MORPHINE, OXYCODONE AND FENTANYL PRESCRIBING PATTERNS IN THE LOCAL HEALTH AUTHORITY OF MESSINA, ITALY}

doi:10.1136/ejhpharm-2013-000276.313

${ }^{1} \underline{\text { S Coppolino, }}{ }^{2} \mathrm{C}$ Sgroi. ${ }^{1}$ P.O. Barone I. Romeo, U.O.S. Farmacia, Patti (Messina), Italy; ${ }^{2}$ ASP Messina, Dipartimento del Farmaco, Messina, Italy

Background Pain is associated with significant economic, social and health impact. The cost of uncontrolled pain is enormous, both to individuals and to society as it leads to a decline in quality of life and disability. Several publications and guidelines stress the efficacy and safety of opioid-based treatment for cancer and non-cancer pain management. Until recently Italian legislation was very restrictive concerning the use of opioids, making Italy one of the lowest users of medical opioids in Europe. In 2010 law no. 38 eased the prescription of opioids for cancer and non-cancer pain.

Purpose To evaluate the incidence and cost of using morphine (N02AA01), oxycodone (N02AA05) and fentanyl (N02AB03) in order to assess prescribing patterns in the Local Health Authority (LHA) between 01/01/2010 and 30/09/2012.

Materials and Methods Data were collected from 'Farmanalisi.it' database which records all prescriptions reimbursed by the Messina LHA. All consumption data were expressed in a standardised way and costs as direct costs to the LHA and recorded on a data sheet.

Results During the 2010-2011 period the consumption of morphine (os/IV) remained substantially stable (1,763 units in $2010 \mathrm{vs.}$ 1,730 in 2011). By contrast, consumption of oxycodone and transdermal fentanyl rose (for oxycodone 3,742 units in 2010 vs. 3,888 in 2011; for transdermal fentanyl 13.680 units in 2010 vs. 13,881 in 2011). The same trend was recorded for the first nine months of 2012 with 1,600 units for morphine, 2,236 units for oxycodone and 7,499 for transdermal fentanyl. For the LHA, in the evaluated period, direct costs of transdermal fentanyl were higher $(978,428.11 €)$ than the value reported for oxycodone $(180,042.89 €)$ and morphine $(46,279.96 €)$.

Conclusions Data obtained confirm that, in the Messina LHA, many patients received transdermal fentanyl as a first option although it is recommended only when oral morphine is inadequate. This data could allow an evolution of strategies adopted to control 